\title{
Using Empirical Mode Decomposition Scheme for Helicopter Main Gearbox Bearing Defect Identification
}

\author{
Fang Duan \\ School of Engineering \\ London South Bank University \\ London, SE1 0AA, UK \\ duanf@1sbu.ac.uk
}

\author{
David Mba \\ School of Engineering \\ London South Bank University \\ London, SE1 0AA, UK \\ mbad@1sbu.ac.uk
}

\begin{abstract}
Vibration sensors for helicopter health and condition monitoring have been widely employed to ensure the safe operation. Through the years, vibration sensors are now commonly placed on helicopters and have claimed a number of successes in preventing accidents. However, vibration based bearing defect identification remains a challenge since bearing defects signatures are usually contaminated by background noise resulting from variable transmission paths from the bearing to the receiving externally mounted vibration sensors. In this paper, the empirical mode decomposition (EMD) scheme was utilized to analyze vibration signal captured from a CS29 Category ' $A$ ' helicopter main gearbox, where bearing faults were seeded on one of the planetary gears bearing of the second epicyclic stage. The EMD scheme decomposed vibration signal into a number of intrinsic mode functions (IMFs) for subsequent envelope analysis. The selection of appropriate IMFs to characterize bearing fault signatures was discussed. The analysis result showed that the bearing fault signatures were successfully characterized and revealed the efficacy of the EMD scheme.
\end{abstract}

Keywords- helicopter main gearbox; fault diagnosis; empirical mode decomposition

\section{INTRODUCTION}

Helicopter main gearbox (MGB) converts high rotation speed gas turbines input to low speed, high torque to drive main rotor blades and related to subsystems, e.g. lubrication and generator. MGB suffers from high temperature and stress resulting from a significant amount of frictional heat generated within MGB. Unlike jet aircraft, helicopter does not have redundant transmission system and hence the malfunction of the MGB can cause serious disaster. Although Health and Usage Monitoring Systems (HUMS) has been widely installed in helicopters to monitor the operational condition of critical parts, there are still several incident reports as a result of undetected faults of MGB [1].

The one of the major reasons is that sensors are usually installed on casing to avoid complex wiring within MGB. The indirect measurement could not provide accurate information since the signal might be significantly attenuated and/or contaminated. To address this issue, wireless transmission and advanced signal processing methods have been proposed in

The research in conducted under the project contract: European Aviation Safety Agency (EASA), EASA.2012.OP.13, entitled "VHM - Vibration health or alternative monitoring technologies for helicopters". literature. A wireless helicopter MGB condition monitoring system has been design by $\mathrm{Mba}$ and colleagues [2]. In the system, acoustic emission (AE) sensor was attached on the planetary gears to avoid signal attenuation and contamination. AE signal was wirelessly transmitted by two coils, moving coil and stationary coil. The bearing outer race defect (ORD) frequency and its harmonic were successfully detected at the cost of additional hardware.

The typical wear-out or defect of components might result in the increased vibration amplitude and appearance of feature frequencies. Therefore, vibration sensors have been well developed and exploited for the purpose of machinery health monitoring. Many diagnosis approaches are proposed to detect faults related frequencies under the challenge of strong background noise. The envelope analysis has been proven to be a powerful tool for detecting bearings and gearboxes defects $[3,4]$. Faults can be inspected by identifying frequencies of the impacts, which results from defects excited resonance. In this method, a band-pass filter has to be utilized to obtain envelope signal. The selection of central frequency and frequency interval has a big impact on diagnosis results. Huang et al. proposed an iterative method, the empirical mode decomposition (EMD) scheme, which can decompose a multicomponent signal into a number of intrinsic mode functions (IMFs) [5]. Each IMF represents a mono-component function versus time. The EMD scheme has gained popularity for dealing non-linear and non-stationary signals in many fields, such as acoustic, biological, ocean, earthquake, climate and faults diagnosis [6].

The aim of this paper is to use available vibration signal to detect MGB faults using EMD. Since vibration sensors are already placed at key locations to monitor the operational condition in the current HUMS, there is no need of additional hardware. The remaining paper is organized as follows. Section II describes the experiment rig. Experimental results and EMD scheme are discussed in Section III. The findings of the study are concluded in Section IV. (PHM-2016 Chengdu) 


\section{EXPERIMENTAL RIG}

A CS29 Category 'A' SA 330 Puma helicopter MGB was mounted on test bench with accelerometers (PCB 352C03, sensitivity of $10 \mathrm{mV} / \mathrm{g}$ ) attached to the case of the gearbox, as shown in figure 1 . The locations of accelerometers (indicated as red dots in figure 1) are replicable with HUMS setup. The accelerometers were connected to a NI cDaq 9188-XT data acquisition chassis and signals were acquired at $51.2 \mathrm{kHz}$ sampling rate.

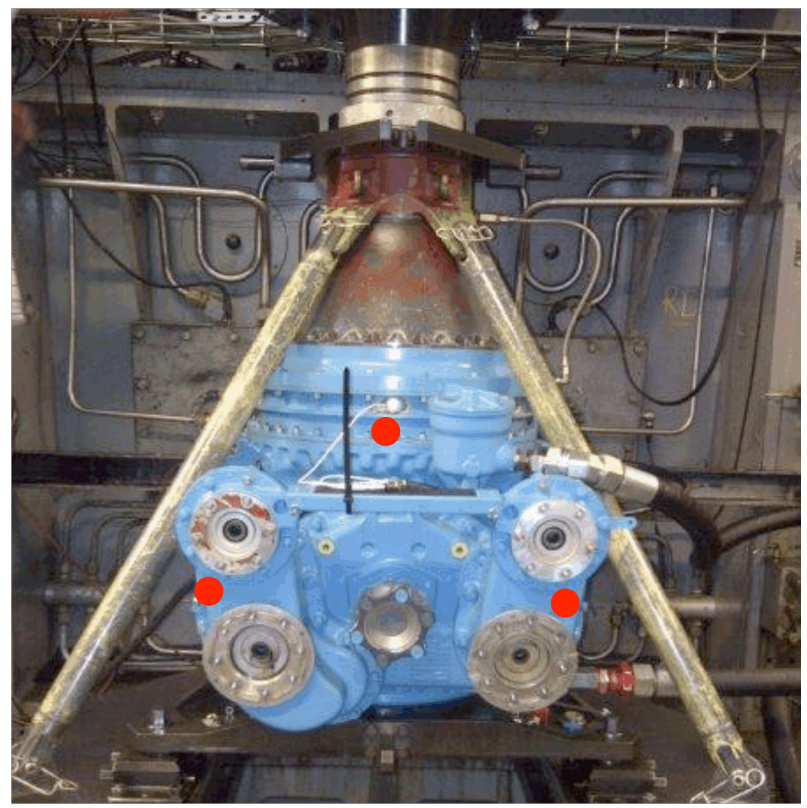

Figure 1. MGB mounted on test bench. Three dots show the location of vibration sensors.

The MGB consists of five reduction gear modules (RGMs), left hand $(\mathrm{LH})$ and right hand $(\mathrm{RH})$ forward (Fwd) RGMs, after (Aft) RGM, main RGM and 2-stage epicyclic (Epi) RGM, as shown in figure 2 . The defect was seeded on one of the planetary gears bearing of the second epicyclic stage, which is shown in figure 3(a). The slightly damage was simulated by machining a rectangular slot of $10 \mathrm{~mm}$ wide and $0.3 \mathrm{~mm}$ deep across the bearing outer race, as shown in figure 3(b). Figure 3(c) shows the natural spalling around half of the circumference in the inner race.

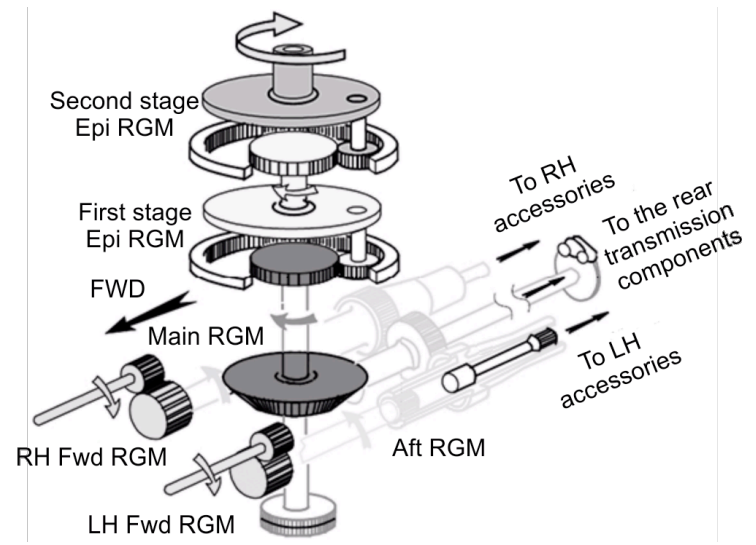

Figure 2. The sketch of helicopter MGB internal parts

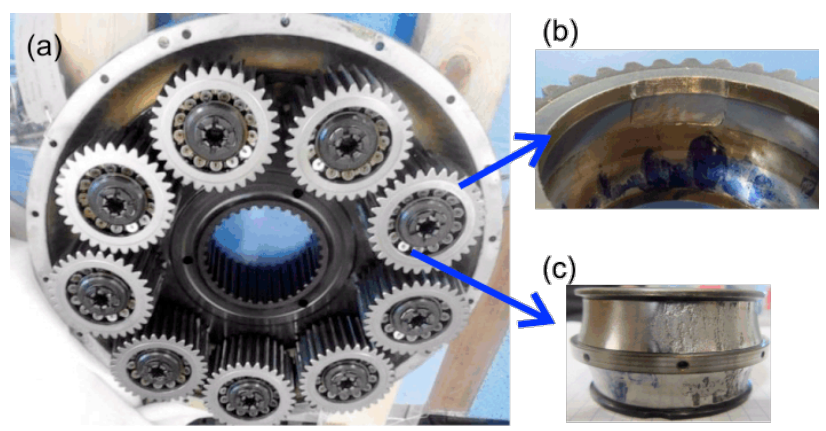

Figure 3. (a) Second stage epicyclic gears;

(b) Slot across the bearing outer race; (c) Inner race natural spalling

\section{EXPERIMENTAL RESULTS AND DISCUSSION}

The experiment was conducted under $110 \%$ of maximum take-off power $(1760 \mathrm{~kW})$ and rotor speed of $265 \mathrm{rpm}$. The equal input torque of LH and RH Fwd RGMs is $368 \mathrm{Nm}$. The bearing outer race defect (ORD) frequency $f_{O R D}$ and inner race defect (IRD) frequency $f_{I R D}$ can be calculated using the standard textbook equations

$$
\begin{aligned}
& f_{\text {ORD }}=\frac{N}{2} \frac{S}{60}\left(1-\frac{d}{D} \cos \alpha\right), \\
& f_{I R D}=\frac{N}{2} \frac{S}{60}\left(1+\frac{d}{D} \cos \alpha\right),
\end{aligned}
$$

where $N=13$ is the number of rollers, $S=1119 \mathrm{rpm}$ is planet gear speed of the second stage Epi RGM, $d=12.5 \mathrm{~mm}$ is the diameter of roller, $D=63.65 \mathrm{~mm}$ is the pitch diameter and $\alpha=0$ is the nominal contact angle. The calculated $f_{O R D}$ and $f_{I R D}$ are equal to $97.42 \mathrm{~Hz}$ and $143.96 \mathrm{~Hz}$, respectively.

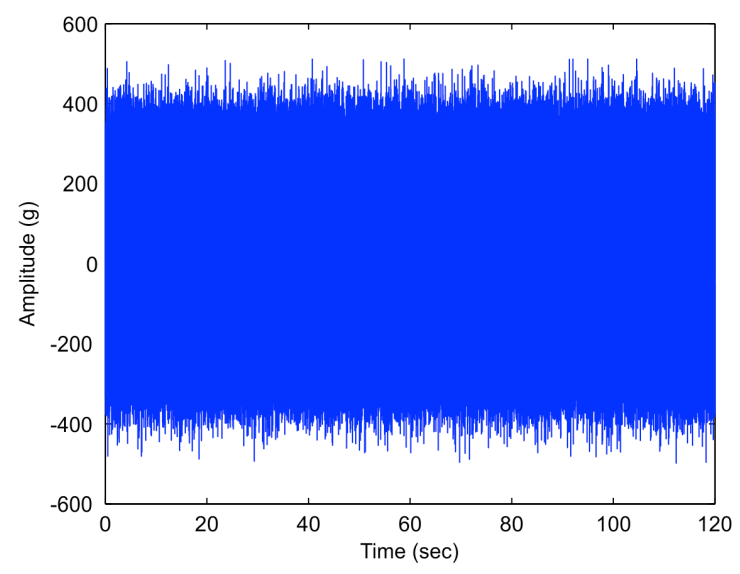

Figure 4. Vibration data from fault bearing of the second epicyclic stage in time domain

The recorded vibration signal with the largest amplitude was utilized to conduct signal processing. Figures 4 and 5 show the recorded vibration signal in time and frequency domain, respectively. The frequency domain spectrum was obtained by using fast Fourier transform (FFT). In the zoomed in picture of 
figure 5, there was not obvious frequency component around the ORD frequency of $97.42 \mathrm{~Hz}$. The frequency component was masked by the background noise. Since the interested frequency components are located in the low frequency band, a low-pass filter is utilized to remove high frequency components above $1 \mathrm{kHz}$. Then, the filtered signal was decomposed to several IMFs using EMD scheme.

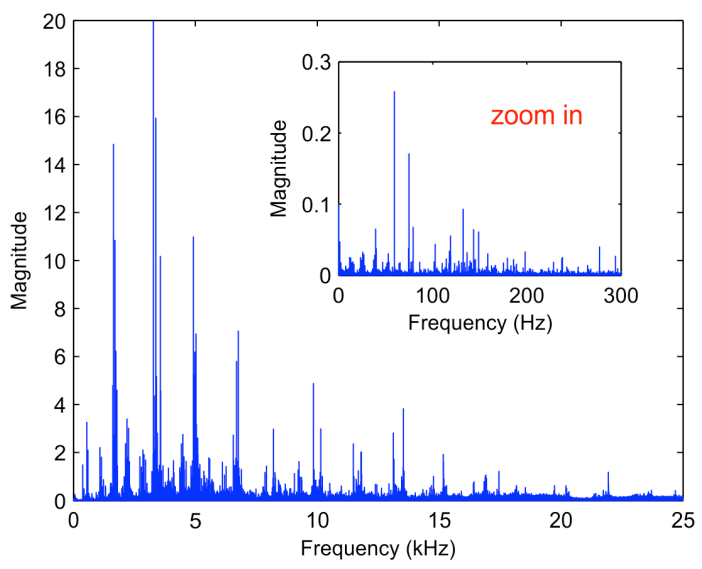

Figure 5. Vibration data from fault bearing of the second epicyclic stage in frequency domain

The EMD scheme is able to decompose non-linear and nonstationary data in to IMFs. Each IMF must satisfy two requirements [5]: (1) In the whole data set, the number of extrema and the number of zero-crossings must either be equal or differ at most by one; (2) At any point, the mean value of the envelope defined by the local maxima and the envelope defined by the local minima is zero. This definition ensures a wellbehaved Hilbert transform of the IMF.

The essential of EMD is to identify the intrinsic oscillatory modes by their characteristic time scales in the data empirically and then decompose the data accordingly [5]. The decomposition procedure is a shifting process, which serves two purposes, to eliminate riding waves and to make the waveprofiles more symmetric. The shifting process is briefly described as follows

(1). Identify all local extrema in the test data $X(t)$.

(2). Connect all the local maxima by a cubic spline line as the upper envelope $U(t)$.

(3). Use the same procedure for the local minima to produce the lower envelope $L(t)$.

(4). Compute the local mean

$$
M(t)=[U(t)+L(t)] / 2
$$

(5). Subtract $M(t)$ from $X(t)$, i.e. $X(t)-M(t)=h_{l}(t)$. If $h_{l}(t)$ satisfies the requirements of IMF, $h_{l}(t)$ is recorded as $c_{l}(t)$.

(6). By repeating the shifting process, $X(t)$ can be decomposed into $N$ IMFs and a residue, $r(t)$, i.e.

$$
X(t)=\sum_{i=1}^{n} c_{i}+r_{n}
$$

The shifting process has to be iterated several times until reaches stop criteria, i.e. the component $c_{n}$ or the residue $r_{n}$ become too small.

The vibration signal was decomposed into several IMFs based on the process described in the previous paragraph. Figures 6 and 7 show the waveform of decomposed IMFs 1-8 computed by EMD. The corresponding spectra of IMFs are shown in figures 8 and 9. Only 8 IMFs are shown in these figures because the low frequency IMFs are less interested in this study, which are neglected. The feature frequencies $f_{O R D}$ $97.42 \mathrm{~Hz}$ of outer race defect and $f_{I R D} 143.96 \mathrm{~Hz}$ of inner race defect are clearly characterized in IMF4 and IMF3 of figure 8, respectively. The same signal processing was applied to the recorded vibration signal under healthy gear condition. The decomposed IMFs 1-4 are shown in figure 10. The feature frequencies $f_{O R D}$ and $f_{I R D}$ are not shown in the figure.

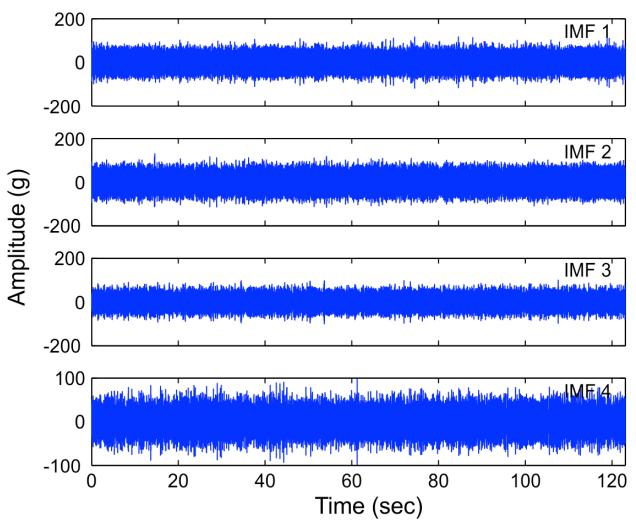

Figure 6. The calcuated IMFs 1-4 under defect condition

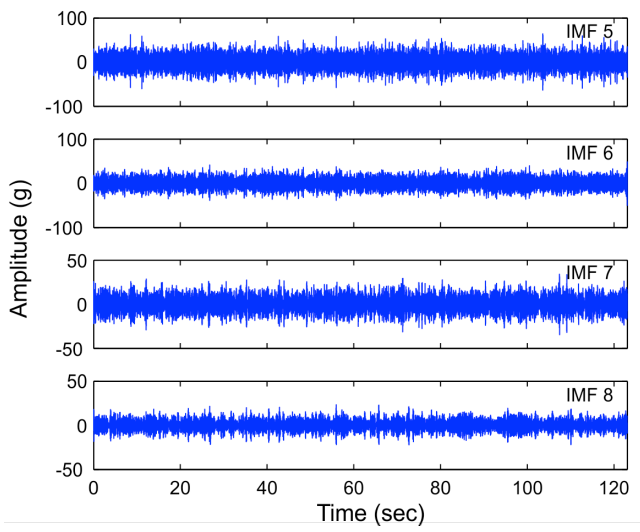

Figure 7. The calcuated IMF 5-8 under defect condition 


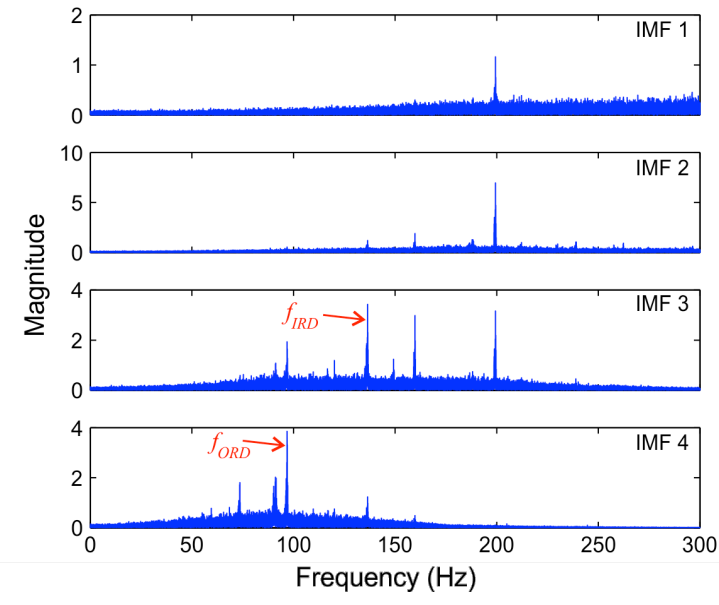

Figure 8. The Fourier spectra of calcuated IMFs 1-4 under defect condition

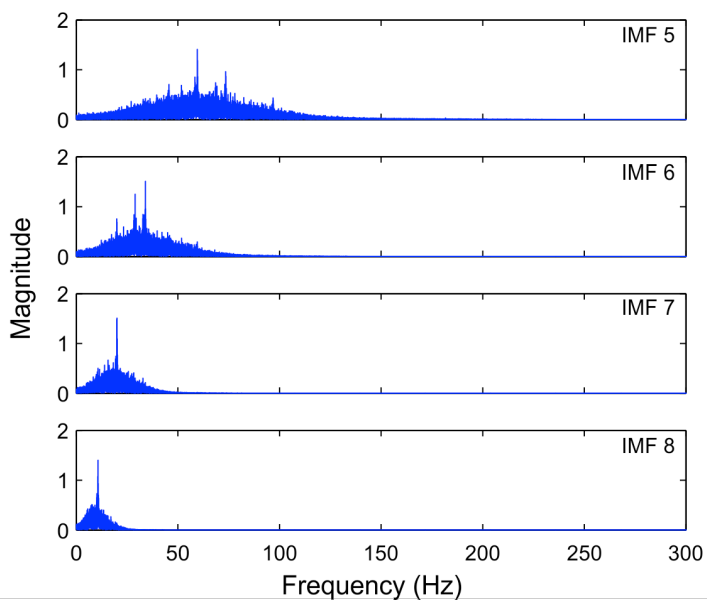

Figure 9. The Fourier spectra of calcuated IMFs 5-8 under defect condition

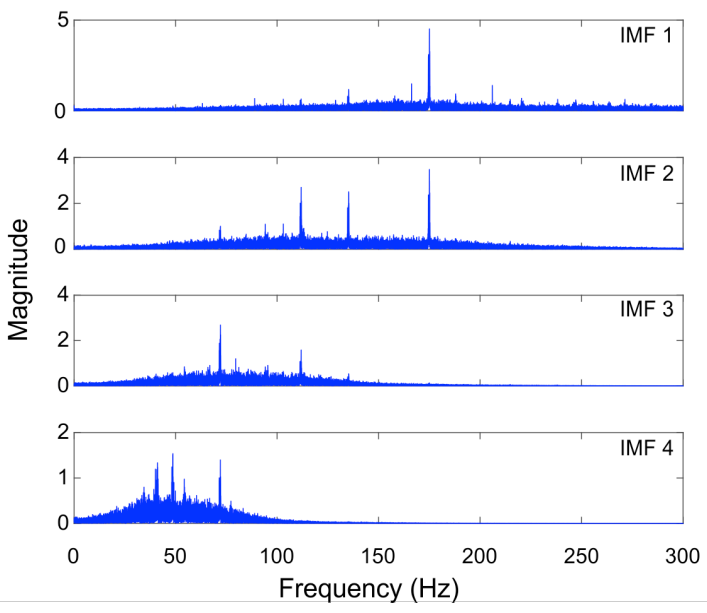

Figure 10. The Fourier spectra of calcuated IMFs 1-4 under healthy condition

\section{CONCLUSION}

In the study, vibration signal was utilized to detect bearing outer and inner race defects of a planetary gear bearing of the second epicyclic stage in a helicopter MGB. Since the vibration signal was recorded by vibration sensors attached on the case of a helicopter main gearbox, the feature frequencies were masked by background noise in the vibration signal frequency spectrum obtained using standard FFT. After filtered by lowpass filter, the vibration signal was decomposed to IMFs using EMD scheme. The feature frequencies $f_{O R D}$ of outer race defect and $f_{I R D}$ inner race defect were clearly observed in Fourier spectra of IMFs. It has been shown the effectiveness of EMD in characterizing features frequencies.

\section{REFERENCES}

[1] Department for Transport, "Report on the accident to aerospatiale (Eurrocopter) AS332 L2 Super Puma, registration G-REDL $11 \mathrm{~nm}$ NE of Peterhead Scotland, on 1 April 2009," 2011.

[2] F. Elasha, M. Greaves, D. Mba and A. Addali, "Application of Acoustic Emission in Diagnostic of Bearing Faults within a Helicopter Gearbox", Proceedings of the 4th International Conference on Through-life Engineering Services, vol. 38, 2015,

[3] W. Wang, "Early detection of gear tooth cracking using the resonance demodulation technique", Mechanical Systems and Signal Processing, vol. 15, no. 5, pp. 887-903, 2001.

[4] R. Randall and J. Antoni, "Rolling element bearing diagnostics - A tutorial", Mechanical Systems and Signal Processing, vol. 25, no. 2, pp. 485-520, 2011.

[5] N. Huang, et al. "The empirical mode decomposition and the Hilbert spectrum for nonlinear and non-stationary time series analysis" Proc. $\mathrm{R}$ Soc. London, vol. 454, pp.903-995, 1998.

[6] N. Huang and S. Shen, "Hilbert-Huang Transform and Its Applications" Singapore: World Scientific, 2005. 